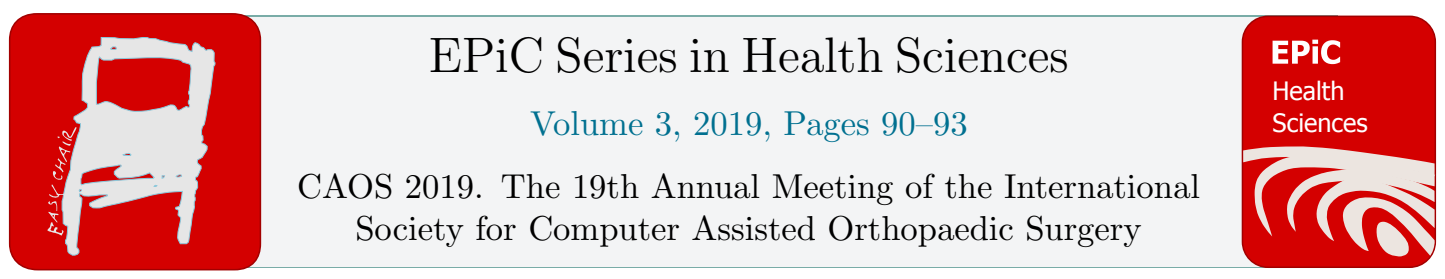

\title{
Early Experience with CAOS Enhanced Total Knee Arthroplasty - A Global, Multi-surgeon Evaluation
}

\author{
Yifei Dai ${ }^{*}$, Valerie Bauler ${ }^{1}$, Stephan Delpech ${ }^{2}$, Ian Gradisar ${ }^{3}$, Phillip \\ Lewandowski ${ }^{3}$, Jefferson Craig Morrison ${ }^{5 *}$ \\ ${ }^{1 *}$ Exactech Inc, Gainesville, FL 32653, USA \\ ${ }^{2}$ Hosptial Prive Marseille Vert Coteau, Marseille, FR \\ ${ }^{3}$ Crystal Clinic Orthopaedic Center, Akron, OH \\ ${ }^{4 *}$ Southern Joint Replacement Institute, Tristar Centennial Hospital, Nashville, TN \\ yifei.dai@exac.com, craig@sjri.com
}

\begin{abstract}
This study investigated surgeons' perceptions of their experience while adopting a novel CAOS enhanced mechanical instrument system for TKA, including ease of usage, task complexity, and demands of surgical time and physical activity. A group of 9 surgeons from multiple countries with no experience in the investigated system used the CAOS enhanced mechanical instrument system during their surgical practice. After performing each TKA case, the surgeon independently completed a 6-section questionnaire formulated to survey his/her experience with the case. The results demonstrated high level of surgeons' experiences with the adoption of the CAOS enhanced conventional instrumentation, with particularly satisfying experiences in minimum demand of time in landmark acquisition and disruption of the existing surgical process.
\end{abstract}

\section{Introduction}

Computer-assisted orthopaedic surgery (CAOS) offers increased accuracy and precision to the bony resections compared to the conventional techniques [1]. Despite the proven benefits provided by CAOS technology, one of the drawbacks for its adoption by the surgeons may be the inconvenience of switching from conventional instruments to CAOS-specific instruments. Recently a novel system has been introduced to enhance conventional mechanical instruments with CAOS technology, removing the need for significant instrument change. The system has been demonstrated to offer improved accuracy from conventionally instrumented surgeries and require a minimum duration of learning in 
previous lab studies [2,3]. Extended investigation on actual TKA cases is needed to confirm the promising results under clinical setting.

To date, limited studies have systematically assessed the early experiences from the surgeons while adopting a new surgical technology. While existing investigations often gauge a surgeon's early experience with measurements such as surgical time and patient outcomes in the initial series, a focus on the operating room is also needed as it is a multifaceted environment that exposes operating surgeons to considerable stress-inducing conditions that may impact their surgical performance [4]. This situation may be particularly magnified during the learning of a new technology.

To evaluate whether adding CAOS guidance to the existing conventional instruments offers seamless transition from surgeons' familiar conventional surgery, the purpose of this study was to measure the early adoption experience from the surgeons' perspectives with the CAOS enhanced conventional instrumentation under clinical setting.

\section{Materials and Methods}

A group of 9 surgeons from 3 geographic regions (France, Australia, United States) highly experienced and predominately or exclusively working with conventionally instrumented TKA were studied. None of the surgeons had used the studied technology previously. Each surgeon performed primary TKA surgeries following their usual surgical techniques except for the addition of CAOS guidance that integrated with the conventional cutting blocks.

After every case, each surgeon independently completed a survey with 12 -item questionnaire (6 for tibia and 6 for femur) covering multiple dimensions of his/her experience during that case regarding bony preparation of the tibia and femur. The specific dimensions assessed were: 1) Over all ease of use; 2) Demand of surgical time: Speed of Landmark Acquisition; 3) Task complexity and physical demand: CAOS Guided Resection Parameter Adjustment Range, Ease of CAOS Guided Resection Adjustments, Stability of Cutting Blocks, and Integration with Existing Surgical Process. Each experience questionnaire was graded in 6 levels from the highest to the lowest as: "excellent", "very good", "good", "neutral", "poor", and "very poor". The surveys were analyzed for the distribution of experience level in each question. The top two rated experience categories were identified among the assessed categories.

\section{Results}

A total of 36 TKA cases were performed by the 9 surgeons using CAOS enhanced conventional instrumentation. The number of cases performed by each surgeon ranged from 1 to 9 . High levels of surgeons' rating were shown in the summary of grading results, with over $94 \%$ of the total case experiences being "good" or better in all categories for the bony preparations of both femur and tibia (Fig 1). More than one third of the case experiences were reported as "excellent" in any given category. The top two surgeon experiences were "Speed of Landmark Acquisition" and "Integration with Existing Surgical Process" among all categories, with more than 50\% of the case experiences as "excellent", and over 91\% with "excellent" or "very good" (Fig 2). 

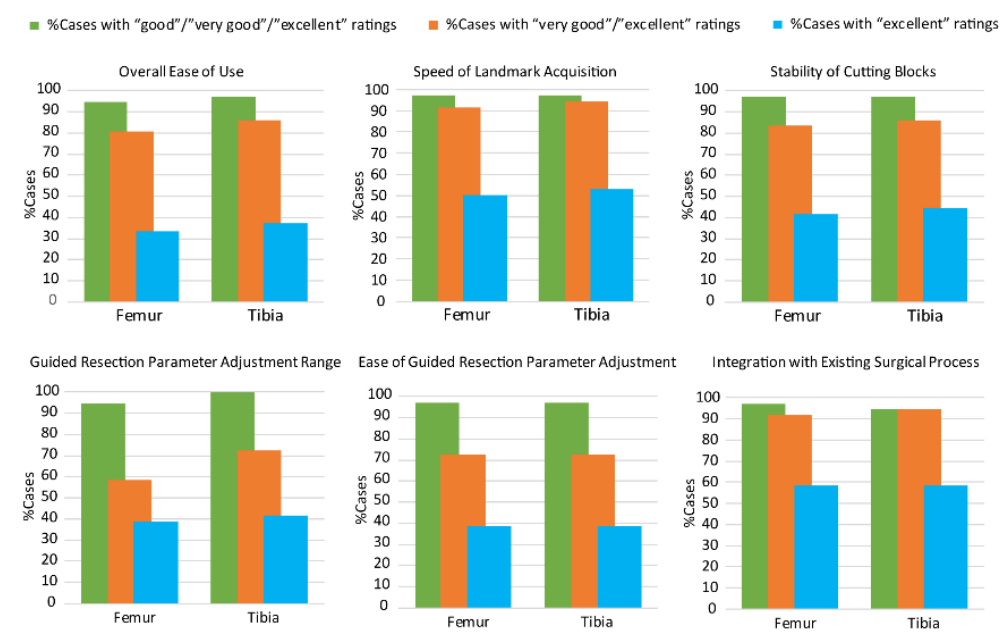

Figure 1. \% cases in each experience category surveyed from the 9 surgeons.
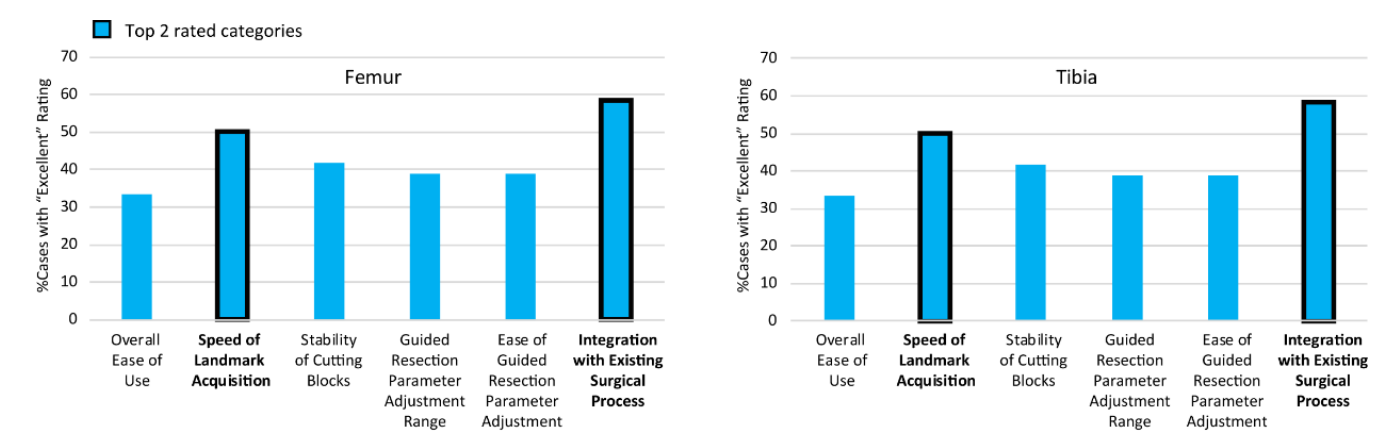

Figure 2. Comparison of "excellent" ratings in femoral and tibial categories, with the two highest rated categories highlighted.

\section{Discussion}

The study employed a multi-dimensional assessment of the early experiences with a CAOS enhanced conventional instrumentation technology from a multi-national group of surgeons. The results demonstrated high levels of surgeons' experience during the introduction of CAOS guidance to the existing conventional instruments. The technology studied was demonstrated to provide an easy to adopt solution for adding the proven benefit of CAOS technology to conventional surgeries without being a stress or demanding point for the surgeons. The two top-rated categories reflected the advantages of the technology that minimizes surgical time extension introduced by landmark acquisition and avoids disruption of the existing surgical process.

When it comes to embracing a new surgical technology, it is unquestionably important to assess the objective measures of the impact on operating room efficiency (surgical time), as well as patients' safety and outcomes. However, the field of surgery has been slow to acknowledge the impact of surgeons' experiences on intraoperative stress, complexity, and physical demands on surgical performance [4], which needs to be assessed with the introduction of advanced surgical technology, especially when it is developed to address the learning hurdle while adding the benefits of CAOS guidance to conventionally instrumented surgery [5-8]. 
This investigation may inform future study design of assessment of surgeons' experience with learning new orthopedic devices and surgical technologies, potentially taking into considerations of other cofounding factors associated with subjective measurements during the adoption phase.

\section{References}

[1] Sparmann M, et al. Positioning of total knee arthroplasty with and without navigation support: a prospective, randomised study. J Bone Joint Surg Br. 2003;85(6):830-5.

[2] Dai Y, et al. Learning of a CAOS enhanced mechanical instrument system for total knee arthroplasty: a CUSUM analysis. CAOS 2018.

[3] Dai Y, et al. Accuracy of a CAOS enhanced mechanical instrument system for total knee arthroplasty. CAOS 2018

[4] Arora S, et al. The impact of stress on surgical performance : a systematic review of the literature. Surgery. 147:318-30.

[5] Brin YS, et al. Imageless computer assisted versus conventional total knee replacement. A Bayesian meta-analysis of 23 comparative studies. Int Orthop,35(3):331-9,2011.

[6] Hetaimish BM, et al. Meta-analysis of navigation vs conventional total knee arthroplasty. J Arthroplasty,27(6):1177-82,2012.

[7] Schnurr C, et al. Influence of computer navigation on TKA revision rates. Int Orthop. 2012;36(11):2255-60.

[8] Schnurr C, et al. The Effect of Computer Navigation on Blood Loss and Transfusion Rate in TKA. Orthopedics. 2010; 13;33(7):474. 